\title{
CONTRIBUIÇÃO AO ESTUDO DAS AFECÇÕES PULMONARES NAS AUTÓPSIAS PEDIÁTRICAS*
}

\author{
CONTRIBUTION TO THE STUDY OF PULMONARY PEDIATRIC PATHOLOGY IN AUTOPSIES
}

\author{
Luiz C. Peres ${ }^{1}$; Fábio A. Moraes² \& César M. Yukita²
}

\begin{abstract}
${ }^{1}$ Docente do Departamento de Patologia; ${ }^{2}$ Aluno do Sexto Ano do Curso de Ciências Médicas. Faculdade de Medicina de Ribeirão Preto da Universidade de São Paulo.

Correspondência: Prof. Dr. Luiz César Peres, Departamento de Patologia da Faculdade de Medicina de Ribeirão Preto da Universidade de São Paulo, Ribeirão Preto - SP, CEP: 14049-900, Fone: 016602 3072, fax: 016633 1068, e-mail: Icperes@fmrp.usp.br
\end{abstract}

PERES LC; MORAES FA \& YUKITA CM. Contribuição ao estudo das afecções pulmonares nas autópsias pediátricas. Medicina, Ribeirão Preto, 32: 303-315, jul./set. 1999.

RESUMO: Modelo do estudo: estudo retrospectivo. Objetivos: determinar a freqüência de algumas das principais afecções pulmonares, pediátricas, com base na casuística de autópsias do Serviço de Patologia do HCFMRP-USP, comparando-a com a literatura, a fim de produzir um texto com informações atualizadas, que possa servir de orientação ao estudo dos problemas, principalmente para alunos de graduação. Metodologia: foram analisados os seguintes dados de quinhentas e cinco (505) autópsias pediátricas, realizadas no Serviço de Patologia do HCFMRP-USP, entre junho de 1993 e junho de 1996: sexo, idade gestacional, idade no momento da morte, peso corporal, causa da morte, peso e alterações pulmonares (petéquias, hemorragias, defeitos de lobação, hipoplasia, pneumonia, pneumotórax, hemotórax, derrame pleural, enfisema intersticial, doença da membrana hialina, displasia broncopulmonar e atelectasia). 0 programa Epi-Info, versão 6, foi usado para a tabulação dos dados e análise estatística, e os resultados, comparados com a literatura. Resultados: o sexo masculino predominou $(53,7 \%)$ sobre o feminino $(45,9 \%)$ e indeterminado(0,4\%). A maior parte do óbitos ocorreu no período neonatal precoce $(47 \%)$, intra-uterino $(19 \%)$ e neonatal tardio $(7 \%)$, somando $73 \%$ de todos os casos. A idade gestacional variou de dezesseis (16) a quarenta e três) 43 semanas. Prematuridade foi a causa de morte mais freqüente $(29,7 \%)$, seguida pelas anomalias congênitas (10,9\%), anoxia intra-uterina $(8,3 \%)$, infecções $(8,1 \%)$, causas perinatais $(2,4 \%)$, isoimunização $(1,8 \%)$, neoplasias $(0,4 \%)$ e outras $(38,4 \%)$. As afecções pulmonares mais comuns foram: petéquias $(49,1 \%)$, hemorragia maciça $(34,5 \%)$, derrame pleural $(29,5 \%)$, doença da membrana hialina $(25,6 \%)$, atelectasia $(24,6 \%)$, pneumonia (16,8\%), pneumotórax $(10,8 \%)$, lobação anormal $(8,6 \%)$, enfisema intersticial (5\%), hipoplasia $(4,4 \%)$ e displasia broncopulmonar $(2,4 \%)$. Conclusões: as afecções pulmonares são muito freqüentes nas autópsias pediátricas, representando, muitas vezes, a causa da morte e estão, de uma forma geral, de acordo com as freqüências encontradas na literatura, havendo, no entanto, algumas variações que, possivelmente, refletem diferenças na atenção pré e perinatal, nos diferentes países. As alterações pulmonares identificadas estão, em sua maioria, relacionadas à prematuridade e anomalias congênitas, que representam um terço de todos os óbitos, constituindo-se nos grupos cuja redução traria os maiores benefícios médicos e sócio-econômicos.

UNITERMOS: Pneumopatias. Pediatria.

*Trabalho realizado no Departamento de Patologia da Faculdade de Medicina de Ribeirão Preto da Universidade de São Paulo, Ribeirão Preto - SP. 


\section{INTRODUÇÃO}

As autópsias pediátricas correspondem a cerca de $22 \%{ }^{(1)}$ de todas as autópsias realizadas no HCFMRP-USP, por ano. Dentre estas, $63 \%$ ocorrem no período perinatal e $54 \%$ no período neonatal. Os problemas pulmonares são de grande importância, principalmente nos casos neonatais, pois, muitas vezes, constituem-se na causa da morte. Em adição a este fato, ressalte-se que a prematuridade, principal problema no período perinatal, tem como alteração mais importante a imaturidade pulmonar, responsável, em grande parte, pela asfixia neonatal e pela cadeia de eventos que culminam com a morte. Por outro lado, as anomalias congênitas, muitas vezes, cursam com hipoplasia pulmonar que, da mesma forma que a imaturidade, leva à asfixia neonatal. Crianças nascidas a termo e aquelas que sobrevivem ao período neonatal estão sempre sujeitas às pneumonias, colocando uma vez mais os problemas pulmonares como diretamente relacionados com a morte.

O objetivo do presente artigo é mostrar os principais problemas pulmonares nas autópsias pediátricas em termos de freqüência e relevância, a partir da casuística do Serviço de Patologia do HCFMRP-USP, de modo a subsidiar a abordagem diagnóstica e terapêutica daqueles profissionais que lidam com os fetos e as crianças, bem como servir de orientação aos alunos de graduação.

\section{MATERIAL E MÉTODOS}

Para se determinarem os principais problemas pulmonares foram compilados os dados referentes às autópsias pediátricas, realizadas no Serviço de Patologia do HCFMRP-USP, no período de junho de1993 a junho de 1996. Os dados de interesse, além dos relativos à identificação, foram os seguintes: sexo, idade gestacional, idade no momento da morte, peso dos pulmões, peso corporal, itens da Declaração de Óbitos e toda e qualquer alteração significativa sobre os pulmões, tais como petéquias, hemorragias, defeitos de lobação, hipoplasia, pneumonia, pneumotórax, hemotórax, derrame pleural, enfisema intersticial, doença da membrana hialina, displasia broncopulmonar e atelectasia.

O programa Epi-Info, versão 6, foi usado para a tabulação dos dados e análise estatística.

\section{RESULTADOS E COMENTÁRIOS}

Os comentários serão feitos de acordo com o esquema a seguir, e os dados referentes a números de casos e porcentagens serão aqueles constantes das tabelas que se seguem.

\section{PRINCIPAIS PROBLEMAS PULMORES EM} AUTÓPSIAS PEDIÁTRICAS

I - CONGÊNITOS:
1. Agenesia
- Bilateral
- Unilateral

2. Hipoplasia

3. Isomeria

4. Defeitos de lobação

5. Malformação adenomatóide

II - ADQUIRIDOS

1. Pneumotórax

2. Derrame pleural

3. Pneumonia

4. Doença da membrana hialina

5. Displasia broncopulmonar

6. Hemorragia maciça

7. Hemorragia petequial

8. Enfisema

- alveolar

- intersticial

9. Aspiração

- mecônio

- conteúdo gástrico

10. Atelectasia

\section{DADOS GERAIS}

As causas de morte dos quinhentos e cinco (505) casos autopsiados e analisados no período estão representadas na Tabela I. O coeficiente de mortalidade infantil em Ribeirão Preto, no período estudado, caiu de 20,2 para 16,3 por mil nascidos vivos, sendo que, no ano de 1996, 2/3 dos óbitos ocorreram no período neonatal, tendo como principais problemas as afecções perinatais, correspondendo a $76 \%$ dos casos, seguidas das anomalias congênitas, com $22 \%{ }^{(2)}$. Em nossa casuística, cerca de um terço dos casos teve a morte relacionada à prematuridade e suas conseqüências, revelando a grande importância dela na mortalidade infantil. Muito embora a prematuridade indique imaturidade orgânica geral, é o pulmão o órgão chave, responsável, em última análise, pela pos- 


\begin{tabular}{|lrr|}
\hline \multicolumn{3}{|l|}{ Tabela I - Causas de morte nos casos estudados } \\
\hline Causas de morte & № & \multicolumn{1}{c|}{$\%$} \\
\hline Prematuridade e conseqüências & 150 & 29.7 \\
Anomalias congênitas & 55 & 10.9 \\
Anoxia intra-uterina & 42 & 8.3 \\
Infecções (>2 semanas) & 41 & 8.1 \\
Causas perinatais & 12 & 2.4 \\
Isoimunização & 9 & 1.8 \\
Neoplasias & 2 & 0.4 \\
Outras & 37 & 7.3 \\
Não catalogadas & 157 & 31.1 \\
\hline TOTAL & 505 & 100.0 \\
\hline
\end{tabular}

sibilidade de sobrevida ou não do recém-nascido. Por ter diversas causas, no entanto, a prematuridade apresenta muitas dificuldades para a redução de sua incidência, ocupando ainda o primeiro lugar entre as causas de morte no primeiro ano de vida ${ }^{(3)}$. Segue, em freqüência, o grupo das anomalias congênitas, que, em nossa casuística, foi responsável por mais de $10 \%$ dos óbitos. Quando consideradas todas as anomalias congênitas, no entanto, e não somente aquelas que determinaram a morte, sua frequiência, em nosso serviço, sobe para cerca de um quarto de todas as autópsias fetais e pediátricas ${ }^{(4)}$. Em Ribeirão Preto, as anomalias congênitas já são a principal causa de morte pós-neonatal, no primeiro ano de vida, correspondendo a $26,5 \%$ do total de óbitos nesta faixa etária ${ }^{(2)}$. Deve-se lembrar que muitas das anomalias congênitas são acompanhadas de problemas pulmonares primários ou secundários. Em terceiro lugar, nas causas de morte neste período, vem os casos de anoxia intra-uterina. As causas são também variadas, porém um adequado acompanhamento pré-natal pode identificar muitos dos problemas maternos que levam a ela, tais como hipertensão arterial, diabete melito, infecções, etc., permitindo seu controle e redução da mortalidade. Em quarto lugar, vêm as infecções adquiridas após o período neonatal precoce, que podem ser comunitárias ou hospitalares. Comparando-se com os dados apresentados por Kalousek \& Gilbert-Barness (1997) ${ }^{(3)}$ para os EUA, há aqui uma inversão, pois eles apresentam em terceiro $\left(3^{\circ}\right)$ lugar, as infecções e, em quarto $\left(4^{\circ}\right)$, os problemas perinatais. Esta diferença se faz, possivelmente, em relação ao nosso país; pela melhor atenção pré e perinatal naquele país. Em quinto lugar, estão as causas perinatais não ligadas às anteriores. A isoimunização, em sexto lugar, representa um grupo especial, pois, mesmo com uma freqüência de apenas $1,8 \%$, tem grande importância já que, muitas vezes, denota acompanhamento pré-natal inadequado, quando não inexistente. Na maioria das vezes, a isoimunização, suficiente para determinar problemas clínicos, incluindo hidropsia, ocorre pelo fator $\mathrm{Rh}$, mas não se pode esquecer que algumas delas são relacionadas com o sistema $\mathrm{ABO}$ e, muito raramente, com outros sistemas sanguiíneos. Ressalte-se que a incompatibilidade sangüínea pelo sistema ABO é muito mais comum do que pelo sistema $\mathrm{Rh}$, porém, apenas ocasionalmente, determina repercussões importantes. Aqui também há uma grande discrepância em relação à casuística acima $\operatorname{citada}^{(3)}$, pois, nos EUA, estes problemas estão em décimo $\left(10^{\circ}\right)$ lugar e somente à frente das alterações metabólicas, sendo a justificativa a mesma dos problemas anteriores. Em sétimo lugar, estão as neoplasias, que correspondem apenas a $0,4 \%$ das autópsias pediátricas. Estes números, contudo, não refletem a freqüência deste grupo de afecções na faixa etária pediátrica, mas tão somente o fato de que a maioria destas crianças não é encaminhada para exame necroscópico.

A Tabela II mostra a freqüência dos sexos nos quinhentos e cinco (505) casos analisados. Em somente dois (02) casos, correspondendo a $0,4 \%$, o sexo era indeterminado, necessitando outros exames, além da macroscopia, para sua determinação. Dos restantes, o sexo masculino foi mais freqüente, porém, sabe-se que há uma ligeira predominância no nascimento de meninos sobre meninas e que, por outro lado, há maior número de mortes destes no período neonatal, uma vez que há chance aumentada de prematuridade entre meninos ${ }^{(5)}$.

A idade no momento da morte, está mostrada na Figura 1. Observa-se que, em 54\% dos nativivos, a morte ocorre até vinte e oito (28) dias de vida, sendo que, destes, $47 \%$ ocorrem no período neonatal precoce, ou seja, nos primeiros sete (07) dias de vida, e os

\begin{tabular}{|c|c|c|}
\hline Sexo & № & $\%$ \\
\hline Masculino & 271 & 53.7 \\
\hline Feminino & 232 & 45.9 \\
\hline Indeterminado & 2 & 0.4 \\
\hline Total & 505 & 100 \\
\hline
\end{tabular}


restantes $7 \%$ no período neonatal tardio que vai do oitavo $\left(8^{\circ}\right)$ ao vigésimo oitavo $\left(28^{\circ}\right)$ dia de vida. Se considerarmos os óbitos intra-uterinos, responsáveis por $19 \%$ de todas as autópsias pediátricas, $73 \%$ dos óbitos ocorrem abaixo de um mês de vida, o que revela a importância das causas perinatais na mortalidade infantil. Somente $6 \%$ dos nossos casos foram de crianças acima de doze (12) meses, ou seja, 94\% das autópsias do Serviço de Patologia, no período examinado, correspondem a óbitos de menores de um (01) ano.

A Figura 2 traz a distribuição do número de óbitos com relação à idade gestacional. Nota-se que esta variou de dezesseis (16) a quarenta e três (43) semanas, sendo a média 31,5 semanas. A distribuição é bimodal, havendo dois picos, com vinte e oito (28) e trinta e sete (37) semanas. Abaixo de vinte e duas (22) semanas há poucos casos, pois a maioria deles deve representar abortos, já que, em geral, nascem mortos e têm peso inferior a quinhentos (500) gramas. No outro extremo, estão aqueles acima de quarenta (40) semanas, que também são menos freqüentes e representam um risco menor do que daqueles nascidos antes de trinta e sete (37) semanas.

Os dados da Tabela III indicam a freqüência dos problemas pulmonares observados nestes casos, os quais serão comentados individualmente, a seguir.

\section{I- AFECÇÕES PULMONARES CONGÊNITAS}

\section{Agenesia Pulmonar}

De todos os problemas pulmonares, o mais grave é, sem dúvida, a agenesia bilateral. Esta é uma condi- ção de excepcional raridade, geralmente acompanhada de agenesia traqueal e outras anomalias congêni$\operatorname{tas}^{(6,7)}$, incompatível com a vida, não tendo sido vista em nenhum dos casos analisados. A agenesia unilateral é também rara, porém mais freqüente do que a bilateral. Geralmente, não se observa a formação do brônquio do lado afetado e podem estar associados outros defeitos, sejam respiratórios ou não ${ }^{(7)}$. Considera-se aplasia, quando existe uma porção de tecido indiferenciado na extremidade de um brônquio. A sobrevida dos pacientes afetados é muito comprometida e a maioria das mortes ocorre por problemas da pró- 
pria insuficiência respiratória ou afecções pulmonares que se associam. No período avaliado neste estudo, não houve nenhum caso de agenesia unilateral do pulmão, mas já foram observados três (03) casos em nosso Serviço em anos fora do período estudado.

\section{Hipoplasia pulmonar}

Ao contrário da agenesia, a hipoplasia pulmonar é uma condição bastante comum. Ela, geralmente, é secundária a alguma forma de compressão do pulmão ou da caixa torácica e sempre acompanha os casos de oligoâmnio de longa duração, qualquer que seja a sua causa. A hipoplasia pode ser objetivamente determinada pela diminuição da relação peso pulmonar/peso corporal abaixo de 0,0012:1 e é compreendida como uma interferência no desenvolvimento e crescimento pulmonar, que leva à redução não apenas no peso dos mesmos ${ }^{(8)}$, mas, também, inibe o desenvolvimento de novos espaços alveolares ${ }^{(9 a, 9 b)}$.

A forma bilateral é a mais comum e, geralmente, está associada à seqüência do oligoâmnio, seja qual for a sua causa, tendo sido observada em $4,4 \%$ dos nossos casos (Tabela III). A ausência de líquido amniótico leva a compressão fetal, interferindo com o adequado crescimento e desenvolvimento de diversas estruturas, tais como a mandíbula e os pulmões, bem como determinando a deformação de outras, como mãos, pés, orelhas e cartilagem nasal. A esta restrição de movimentos soma-se a própria ausência do

\begin{tabular}{|c|c|c|}
\hline Patologia & Freqüência & $\%$ \\
\hline Hemorragial petequial & $245 / 499$ & 49.1 \\
\hline Hemorragia maciça & $172 / 498$ & 34.5 \\
\hline Derrame pleural & $147 / 499$ & 29.5 \\
\hline Membrana hialina & $128 / 499$ & 25.6 \\
\hline Atelectasia & $123 / 499$ & 24.6 \\
\hline Pneumonia & $84 / 499$ & 16.8 \\
\hline Pneumotórax & $54(499)$ & 10.8 \\
\hline Lobação anormal & $43 / 498$ & 8.6 \\
\hline Enfisema intersticial & $25 / 499$ & 5.0 \\
\hline Hipoplasia & $22 / 498$ & 4.4 \\
\hline Displasia broncopulmonar & $12 / 499$ & 2.4 \\
\hline
\end{tabular}

líquido amniótico, que está sendo constantemente inspirado pelo feto e representa um outro estímulo mecânico para o crescimento e desenvolvimento pulmonar ${ }^{(10)}$. A própria compressão pulmonar leva ao esvaziamento do líquido secretado pelo epitélio brônquico, o qual é também necessário para promover o crescimento do órgão ${ }^{(10)}$. Quanto mais precocemente se instala o oligoâmnio tanto mais intenso é o grau de hipoplasia. Outra causa importante de hipoplasia bilateral é a que decorre de compressão pulmonar secundária a derrame pleural bilateral de longa duração, hérnia diafragmática e anomalias complexas ${ }^{(11,12,13)}$.

Os casos de hipoplasia unilateral estão relacionados à compressão de apenas um dos pulmões, como acontece nas hérnias diafragmáticas unilaterais, deformidades da caixa torácica e mesmo nas cardiomegalias volumosas.

Do ponto de vista macroscópico, o pulmão hipoplásico apresenta volume e peso diminuídos ${ }^{(8)}$, geralmente associados a atelectasia e aspecto carnoso do parênquima. Pode haver defeitos de lobação associados. Durante a autópsia, pode-se notar a hipoplasia e seu grau através de uma manobra simples, que consiste em retirar o bloco torácico em conjunto com os órgãos cervicais e língua, segurando-se, com uma pinça, pela ponta desta última. Nos casos normais, os pulmões cobrem completamente o coração, porém, nas hipoplasias, os mesmos são curtos, deixando este a descoberto. Mnemonicamente, referimos a este como o teste da minissaia.

Microscopicamente, o pulmão hipoplásico tem, caracteristicamente, uma diminuição no número de alvéolos situados entre os bronquíolos e a pleura. Estudos morfométricos demonstram esta redução de maneira inequívoca, sendo que uma contagem radial de alvéolos, menor ou igual a 4,1, indica hipoplasia ${ }^{(8,14)}$. Se a criança sobreviveu por algum tempo, outras alterações podem surgir, as quais serão consideradas mais à frente.

\section{Isomeria}

As observações anatômicas, realizadas desde a Antigüidade, costumavam relacionar os achados com características fixas, tais como a lateralidade. Desta forma, os diversos órgãos duplos foram denominados direitos e esquerdos, de acordo com a sua topografia usual. No entanto, muitos deles apresentam características próprias que os distinguem independentemente do lado onde estejam, como é o caso dos pulmões. Chamamos de direito àquele que possui três $(03)$ 
lobos e tem brônquio fonte mais perpendicular e esquerdo àquele que possui dois (02) lobos e brônquio fonte mais horizontalizado. Em algumas circunstâncias, os pulmões estão topograficamente invertidos, como no situs inversus, e, mesmo assim, continuamos chamando de direito ao que está à esquerda, e esquerdo, ao que se localiza à direita. Situação mais incomum, porém não rara, é a que se observa nas chamadas seqüências da lateralidade, nas quais ocorre uma duplicação de um dos lados. Se o lado direito for duplicado, então, haverá dois pulmões direitos, portanto isomeria direita, a qual geralmente se acompanha de agenesia esplênica. Na isomeria esquerda, há dois pulmões esquerdos e poliesplenia. Em ambas as situações, há, em geral, cardiopatia congênita com drenagem venosa anômala pulmonar e sistêmica ${ }^{(15 ; 16 ; 17)}$.

O diagnóstico da isomeria pulmonar é, portanto, macroscópico. Não há alterações microscópicas características e, por outro lado, a simples isomeria pulmonar não determina nenhum tipo de problema para quem a tem. No entanto, como ela pode estar acompanhada das outras anomalias mencionadas, a sobrevida é dependente destas últimas, principalmente das alterações cardíacas.

\section{Defeitos de lobação}

A lobação é uma característica morfológica dos pulmões. Em algumas circunstâncias, os pulmões podem apresentar variações nesta lobação, seja para mais ou para menos, situação identificada em $8,6 \%$ dos casos analisados. Estes defeitos, geralmente, acompanham outras anomalias congênitas e por si só não representam um problema com implicações na sobrevida fetal ${ }^{(18)}$. O diagnóstico, portanto, é macroscópico, variando de casos sem nenhuma divisão até outros com cinco (05) ou mais lobos. Do ponto de vista microscópico, não há alteração detectável. Sua importância maior é para o patologista que se depara com tal problema durante a autópsia, indicando-lhe que outras defeitos mais importantes podem estar presentes. Teoricamente, este tipo de defeito pode trazer problemas ao cirurgião que necessite fazer uma ressecção segmentar.

\section{Malformação adenomatóide}

Este é um defeito congênito do pulmão, na sua maioria unilateral, caracterizado pela proliferação de brônquios, formando massa intrapulmonar que pode comprometer todo um lobo. Stocker ${ }^{(19)}$ et al. reconheceu, originalmente três (03) tipos, sendo que, atualmente, há cinco (05) tipos aceitos ${ }^{(20)}$ : Tipo 0, descrito como displasia acinar, é incompatível com a vida e está associado a defeitos cardíacos e hipoplasia dérmica, sendo caracterizado, morfologicamente, por pulmões pequenos e compactos ${ }^{(21)}$; Tipo I tem cistos grandes, em pequeno número, que não contém cartilagem, aliás uma característica de qualquer um dos tipos. A parede destes cistos contém fibras musculares lisas e elásticas e o epitélio é colunar pseudoestratificado ciliado. Entre os cistos grandes, há outros menores e também estruturas alveolares; Tipo II apresenta múltiplos cistos de até $1 \mathrm{~cm}$ de diâmetro, homogeneamente distribuídos, que se comunicam com a árvore brônquica de maneira normal e apresentam epitélio cuboidal ou colunar ciliado e não colunar pseudo estratificado ciliado, como o respiratório; Tipo III tem cistos muito pequenos, menores do que $0,5 \mathrm{~cm}$ de diâmetro, conferindo um aspecto sólido à lesão, motivo pelo qual se retirou o termo cística do nome desta entidade. Histologicamente, os pequenos cistos são revestidos por epitélio cuboidal ciliado e, às vezes, não ciliado, lembrando a fase canalicular de desenvolvimento pulmonar, observado entre dezesseis (16) e vinte (20) semanas de idade gestacional. Por fim, Tipo IV, representado por cistos periféricos de origem acinar, cujo revestimento é feito por pneumócitos tipo I aplanados ${ }^{(13)}$.

\section{AFECÇÕES PULMONARES ADQUIRIDAS}

\section{Pneumotórax}

Pneumotórax é uma ocorrência extremamente comum no período neonatal ${ }^{(22,23)}$, sendo encontrado em $10,8 \%$ dos nossos casos. Em geral, é observado em neonatos, principalmente pré-termos de baixo peso, os quais necessitam de ventilação mecânica. Quanto menor a idade gestacional, maior será a chance de ocorrer um pneumotórax. Este pode ser pequeno, identificado durante a autópsia, quando o corpo submerso em água tem a musculatura intercostal perfurada e poucas bolhas de ar podem ser observadas ou, então, volumosos, levando ao rebaixamento diafragmático e, conseqüentemente, das vísceras abdominais. Neste caso, o pneumotórax é do tipo hipertensivo e pode ser o determinante da morte.

Admite-se que, durante a ventilação mecânica, surgem pequenas lacerações bronquiolares ou alveolares através das quais o ar penetra e disseca o interstício pulmonar, chegando até a pleura e ao espaço pleural, formando um enfisema intersticial pulmonar ${ }^{(13)}$, detectado em $5 \%$ dos nossos casos. Como estes neo- 
natos dependem da ventilação mecânica, a mesma deve ser mantida e a alternativa é drenar o tórax. Esta manobra pode ser eficiente e a criança não mais apresentar o pneumotórax, muito embora não seja isenta de riscos, tais como perfuração do parênquima ${ }^{(22)}$ ou do feixe vasculonervoso, com conseqüente hemorragia maciça, como em um caso por nós observado à autópsia. No entanto, em alguns casos, a persistência da passagem do ar cria uma fístula que pode manter o quadro por longo tempo. De qualquer forma, o ar, no interstício pulmonar, leva à compressão de vasos sangüíneos e linfáticos, contribuindo para aumento da pressão vascular pulmonar e suas conseqüências cardíacas. A cronificação do processo leva ao quadro conhecido como enfisema intersticial persistente ${ }^{(24)}$, cuja macroscopia é a de um pulmão com inúmeras áreas císticas, lembrando um favo de mel. Uma conseqüência possível é a embolia gasosa sistêmica ${ }^{(25)}$.

O pneumotórax hipertensivo aumenta a pressão intratorácica, diminuindo o retorno venoso para o coração. Isto é particularmente importante no encéfalo, pois, em geral, leva à hemorragia da matriz germinativa, que se complica com a intraventricular, o golpe de misericórdia. Quando unilateral, o pneumotórax também determina o desbalanço do mediastino e suas nefastas consequiências.

Fora do período neonatal, o pneumotórax é incomum e tem, mais ou menos, a mesma patogenia daquele observado nos adultos.

\section{Derrame pleural}

Derrame pleural significativo é observado nos casos de hidropisia fetal, seja qual for a sua causa ${ }^{(26,}$ 27a,27b). Nestes casos, o líquido é amarelo-citrino, podendo atingir grandes volumes e, desta forma, levar a compressão pulmonar que, se persistente, determina hipoplasia pulmonar. Esta patogênese, no entanto, tem suscitado polêmica sobre qual seria a causa e qual a conseqüência $^{(28)}$.

As causas da hidropisia fetal são muitas, podendo ser divididas em dois grandes grupos: imunitárias e não imunitárias. As causas imunitárias são, em geral, decorrentes da isoimunização pelo fator $\mathrm{Rh}$, porém podem ocorrer também com o sistema $\mathrm{ABO}$ ou, mais raramente, com outros sistemas sangüíneos, enquanto que as não imunitárias compreendem um número elevado de afecções e condições diversas, das quais citamos as seguintes: cardiopatias congênitas, arritmias cardíacas, anomalias congênitas pulmonares, cromossomopatias, hemoglobinopatias e infec- ções. Dentre as causas infecciosas, merece destaque a infecção pelo Parvovírus B19 ${ }^{(29)}$, que infecta os precursores eritróides, impedindo a maturação adequada da linhagem eritrocítica, com conseqüente anemia.

Nos casos de hidropisia fetal, a sobrevida do neonato está na dependência de todas as alterações que ele apresenta, contribuindo para o óbito a hipoplasia pulmonar, se presente. Muitos destes casos resultam em óbito intra-uterino e são abortados com graus variáveis de maceração.

\section{Pneumonia}

As infecções respiratórias são freqüentes e importantes na patologia pediátrica, estando presentes em $16.8 \%$ dos nossos casos (Tabela I). De particular interesse é a pneumonia congênita ou perinatal, pois pode ter gravidade suficiente para culminar com quadro séptico nos primeiros dias de vida ${ }^{(12)}$.

As pneumonias que surgem no período neonatal podem ser ascendentes, isto é, serem conseqüentes ao crescimento de bactérias, fungos e mesmo vírus presentes no canal de parto, muitos dos quais são comensais do trato gastrintestinal. As bactérias são, geralmente, Gram-negativas, dentre as quais a $E$. coli é a mais comum. No entanto, outras bactérias também podem ser encontradas, tais como os estreptococos $\beta$-hemolíticos do grupo B; Listeria monocytogenes e Pseudomonas aeruginosa, além de outras.

Os estreptococos $\beta$-hemolíticos do grupo B são responsáveis por uma infecção respiratória de rápida instalação e curso fulminante, se não for adequada e rapidamente tratada. A macroscopia dos pulmões, nestes casos, não é característica, podendo eles apresentarem-se congestos e colapsados. Muitas vezes, são acompanhados por derrame pleural, que, em algumas circunstâncias, pode ser fibrinoso. A histologia mostra aspecto variável na dependência do tempo de sobrevida. Edema e congestão com pouco colapso alveolar pode ser visto nos casos de morte rápida. Com sobrevida mais longa pode surgir fragmentação da membrana hialina com grande quantidade de bactérias, atelectasia e intenso exsudato inflamatório polimorfonuclear.

A Listeria monocytogenes determina um quadro clínico característico, representado por instalação de febre elevada na mãe, dois a três dias antes do parto. Segue-se trabalho de parto, geralmente prematuro, com a expulsão de natimorto ou feto que, caracteristicamente, apresenta uma miríade de pequenas nodulações brancacentas, puntiformes, distribuídas por 
todo o corpo, inclusive em órgãos internos. O tratamento materno com antibióticos pode ser eficiente para impedir a morte fetal ${ }^{(30)}$. A histologia revela granulomas com centro composto por debris celulares tanto na placenta quanto nos órgãos fetais, nos quais os bacilos Gram-positivos podem ser identificados pela coloração de Warthin-Starry.

A infecção pela Pseudomonas aeruginosa é, geralmente, adquirida no período pós-natal e, geralmente é observada nos pré-termos que necessitam ventilação demorada e antibioticoterapia. Os pulmões costumam estar pesados e firmes e mostrando pequenos pontos subpleurais ou nas superfícies de corte. A histologia confirma estes abscessos e os microorganismos podem ser identificados pelo Gram.

As infecções intra-uterinas podem ocorrer muito precocemente e levar a outras alterações pulmonares, não necessariamente relacionadas com a morte. PERES et al. ${ }^{(31)}$, identificaram espessamento pleural de extensão e intensidade variável em fetos abortados, cuja idade gestacional média de foi de dezoito (18) semanas. Estes casos, correspondendo a cerca de 3\% de todos os abortos examinados, apresentavam sinais de infecção pregressa ou ativa, que, segundo a interpretação dos autores, poderia ser a responsável pelo desencadeamento da ativação fibroblástica que, em última análise, culminaria com a fibrose pleural.

Além das bactérias, também os fungos podem levar a infecção pulmonar neonatal. Os mais comuns são os do gênero Candida. A infecção congênita se faz pela via ascendente, quando, então, não apenas os pulmões, mas, também, a pele e o trato digestivo podem estar acometidos. A forma adquirida após o nascimento, geralmente, está relacionada com a cateterização venosa prolongada. Caracteristicamente, as lesões macroscópicas da candidíase aparecem como pequenos pontos ou placas esbranquiçadas que, do ponto de vista histológico, mostram, geralmente, pequeno infiltrado inflamatório mononuclear em meio ao qual são vistas formas leveduriformes e pseudo-hifas do fungo.

Outros fungos, tais como Aspergillus, Histoplasma, Criptococcus, Paracoccidioides brasiliensis e Pneumocystis podem ser observados em crianças maiores e apresentam características semelhantes aos do comprometimento de adultos.

Pneumonia viral pode ser congênita ou adquirida. A congênita se dá por contaminação hematogênica e pode ser causada por vírus, tais como Citomegalovírus e Herpes. Das pneumonias virais adquiridas res- saltam em importância as causadas pelo vírus do sarampo, pelos Rhinovírus, Adenovírus, Influenza, Varicella, Echovírus, Parvovírus e Vírus Sincicial Respiratório.

\section{Doença da membrana hialina}

A doença da membrana hialina é um dos problemas mais comuns e mais sérios do período neonatal em pré-termos, porém não restrita a este grupo $^{(32)}$, tendo sido observada em 25,6\% dos nossos casos. Ela surge em conseqüência da insuficiente produção de surfactante pelos pneumócitos tipo II, o que acarreta um esforço respiratório aumentado nestas crianças ao qual se alia uma maior dificuldade na difusão de gases, culminando com hipoxia cada vez mais acentuada.

O surfactante começa a ser produzido em pequena escala por volta da vigésima a vigésima-quarta $\left(20^{\mathrm{a}} / 24^{\mathrm{a}}\right)$ semana, porém só é produzido em grande quantidade a partir da trigésima $\left(30^{a}\right) \operatorname{semana}^{(18)}$. Entende-se, portanto, que o risco de desenvolvimento da membrana hialina é inversamente proporcional à idade gestacional, sendo de $60 \%$ para a vigésima-oitava $\left(28^{\mathrm{a}}\right)$ semana e menor do que $5 \%$ para a trigésimasetima $\left(37^{\mathrm{a}}\right)^{(33)}$. O material a que se convencionou chamar de membrana hialina é constituído, em sua maior parte, por debris celulares do revestimento epitelial, componentes do líquido amniótico, proteínas plasmáticas e fibrina agregados sobre a superfície alveolar. As proteínas plasmáticas, principalmente a fibrina, são decorrentes da lesão do endotélio vascular pela hipoxia e acidose que se instala, permitindo a sua saída para a luz alveolar, onde se misturam com restos celulares do epitélio alveolar.

Além da prematuridade, são particularmente crianças de risco os filhos de mãe diabética e os nascidos por cesariana $^{(34,35,36)}$. Outros fatores associados são: aspiração de mecônio, amniorréxis prematura, asfixia neonatal com anoxia e acidose agudas, hipotermia, infecção intra-uterina, cardiopatia congênita, hipoperfusão pulmonar, tocotraumatismo, eritroblastose fetal, além de fatores imunológicos e neuroendó$\operatorname{crinos}^{(18)}$. A secreção de surfactante depende de uma cadeia complexa de eventos dos quais participam hormônios, principalmente o cortisol, além de insulina, prolactina e tiroxina ${ }^{(37)}$. A insulina tem efeito contrário ao do cortisol e, por isso, os filhos de mãe diabética estão mais propensos a desenvolver a doença. Por outro lado, entende-se facilmente porque a administração de corticosteróides, previamente ao parto, pode auxiliar na redução dos riscos de $\mathrm{DMH}^{(38,39,40)}$. 
Os pulmões apresentam-se macroscopicamente firmes, congestos, carnosos, não aerados e com uma evidenciação maior dos septos lobulares, o que lhes confere aspecto rendilhado. A histologia revela um pulmão imaturo, com poucos alvéolos e septos espessados, grande parte dos quais revestidos pelo material hialino que dá o nome à doença. Acompanham estas alterações outras possíveis consequiências da anoxia.

As medidas terapêuticas, atualmente utilizadas para se impedir o desenvolvimento da DMH, têm contribuído para a rápida recuperação de muitas crianças de muito baixo peso com poucas sequielas ${ }^{(41)}$. Crianças que sobrevivem por tempo mais prolongado, necessitando de ventilação mecânica, podem desenvolver quadro crônico de maior gravidade, representado pela displasia broncopulmonar, descrita a seguir.

\section{Displasia broncopulmonar}

Esta é a mais séria consequiência crônica da DMH tratada por ventilação mecânica de pressão positiva, embora não exclusiva desta entidade. A sua etiopatogenia não está completamente elucidada, mas sabe-se que dela participam: toxicidade ao oxigênio, pressão de ventilação, deficiência de antioxidantes, aumento da permeabilidade epitelial, fluxo sangüíneo através de um ducto arterioso patente ${ }^{(42)}$ e mau funcionamento ciliar do epitélio brônquico ${ }^{(43)}$. Estudos experimentais demonstraram que a exposição ao oxigênio dos pulmões de cobaias recém-nascidas promove aumento dos macrófagos alveolares, que por sua vez atraem polimorfonucleares neutrófilos e ambos secretam elastase, aumentando os danos ao parênquima pulmonar ${ }^{(44)}$. Há, também, redução da $\alpha 1$ antitripsina no lavado brônquico, o que acarreta aumento no funcionamento de enzimas proteolíticas, que, por sua vez, lesam ainda mais o pulmão ${ }^{(11)}$. O edema pulmonar secundário à persistência do ducto arterioso piora este quadro por reduzir a complacência pulmonar e aumentar a resistência ventilatória, exigindo mais ventilação e oxigenação.

A morfologia do pulmão varia de acordo com o tempo de evolução da doença. Classicamente, os pulmões apresentam áreas salientes pela hiperinsuflação circundadas por áreas carnosas e deprimidas secundárias à atelectasia. Muitas vezes, os pulmões apresentam a impressão das costelas em função da expansão das áreas hiperinsufladas. A microscopia desta condição mostra os fenômenos de lesão e contínua reparação em um pulmão em desenvolvimento. São reconhecidas três fases distintas na evolução: fase exsudativa ou reparativa precoce, fase subaguda e fase crônica. A primeira, ainda reversível, ocorre entre uma e duas semanas e se caracteriza por lesão alveolar (membranas hialinas) e bronquiolar (necrose, obstrução e reparação). Na fase subaguda, que ocorre entre duas a quatro semanas, surge fibrose intersticial e periductal, hiperplasia de pneumócitos tipo II, proliferação de capilares intersticiais e de células musculares lisas. Nesta fase, já aparecem ácinos colapsados ao lado de outros hiperinsuflados. A fase crônica, que pode surgir após meses, mostra acentuação da fibrose intersticial e pulmão em favo de mel, além de proliferação de células musculares lisas no interstício e lesão vascular, representada por hiperplasia da camada média e espessamento da íntima de pequenos vasos. A hipertensão pulmonar que se instala pode determinar o desenvolvimento de cor pulmonale crônico. Ácinos colapsados ao lado de outros hiperinsuflados também são identificados ${ }^{(12,42)}$.

Todas estas alterações pulmonares levam à manutenção da ventilação mecânica até que o pulmão apresente desenvolvimento adequado, quando o desmame do ventilador se faz necessário, porém de difícil realização.

\section{Hemorragia maciça}

A hemorragia pulmonar maciça é uma condição vista em recém-nascidos pré-termos, cujas causas podem ser muitas e variadas, tais como: hipoxia ou anoxia neonatal, infecção viral ou bacteriana, edema cerebral, hemorragia cerebral intraventricular, hipotermia, doença hemorrágica do recém-nascido, doença da membrana hialina, hiperamonemia, C.I.V.D. e coagulopatia primária ${ }^{(12,18)}$. Na verdade, parece ser muito mais um edema pulmonar hemorrágico do que propriamente uma hemorragia alveolar ${ }^{(45)}$. De qualquer forma, geralmente, é o episódio final em um pretermo com sérios problemas perinatais.

$\mathrm{O}$ aspecto macroscópico é característico, representado por saída de grande quantidade de sangue pelas narinas e boca, estendendo-se desde os pulmões. Estes ficam acentuadamente vermelhos, pouco aerados e drenam grande quantidade de sangue pela superfície de corte. Hemorragia alveolar difusa é o quadro microscópico dominante, geralmente acompanhada de focos de edema.

\section{Hemorragia petequial}

Petéquias são hemorragias puntiformes que podem aparecer em diversos órgãos em variadas circunstâncias. Em patologia perinatal elas são, geralmente, encontradas nas superfícies de órgãos torácicos 
e podem representar aumento da pressão negativa por inspiração vigorosa, portanto associadas aos quadros de anoxia $^{(46)}$. Desta forma, podem ser encontradas tanto em natimortos quanto naqueles que nasceram vivos e é o principal achado nos casos de síndrome da morte súbita da infância ${ }^{(47)}$.

Admite-se que o aumento da pressão negativa intratorácica promova uma sucção do conteúdo vascular, resultando em pequenos focos de hemorragia. Contribui para esta saída de sangue a lesão endotelial capilar secundária à hipoxia e à acidose. É a somatória destes dois fatores que explica o porquê de serem mais freqüentemente observadas nos órgãos torácicos, mas também permite entender porque podem ser encontradas em outros locais.

É importante destacar que o encontro de petéquias na face e em conjuntivas oculares deve levantar a suspeita de asfixia mecânica criminosa, pois, nestes casos, associa-se um elemento de diminuição do retorno venoso ${ }^{(47)}$.

As petéquias podem aparecer em número variável nos casos de anoxia intra-uterina e perinatal, freqüentemente observadas em prematuros, mas, em geral, não chegam a causar sufusões hemorrágicas. A existência destas últimas pode ter o mesmo significado das petéquias faciais e conjuntivais.

$\mathrm{O}$ aspecto histológico das petéquias é bastante variável, pois podem ser encontradas apenas poucas hemácias no conjuntivo ao redor do capilar até pequenas hemorragias intersticiais.

\section{Enfisema alveolar intersticial}

Esta é uma condição que se acompanha de pneumotórax $^{(48)}$, algumas vezes espontâneo, mas comumente associado à DMH, à aspiração de mecônio, e à displasia broncopulmonar ${ }^{(49)}$, em que se necessita fazer ventilação mecânica de pressão positiva. Resulta da formação de pequenas fístulas alveolointersticiais que mantêm distendidos espaços sem revestimento próprio ${ }^{(49)}$. Ocasionalmente, podem se conectar com vasos linfáticos. A macroscopia é a de um pulmão com inúmeras áreas císticas, lembrando um favo de mel. Tais crianças apresentam uma dependência crônica do respirador e raramente conseguem sobreviver. Embolia gasosa pode ser observada em alguns casos $^{(50)}$, bem como pneumomediastino, pneumopericárdio e pneumoperitônio ${ }^{(49)}$. A forma aguda é vista em 5 a $10 \%$ dos pretermos ventilados por DMH. Na forma persistente, as crianças têm, em geral, mais de uma semana de vida.

\section{Aspiração de mecônio e/ou conteúdo gástrico}

Sabe-se que existem movimentos respiratórios fetais desde muito cedo na gestação ${ }^{(51)}$, mas eles, em geral, não são intensos o suficiente para promover a entrada de escamas córneas e mecônio, quando presente no líquido amniótico, até espaços respiratórios distais. Contribui para este fato a secreção pela mucosa brônquica de líquido com maior viscosidade do que o amniótico que, de certa forma, impede a penetração distal deste e seu conteúdo sólido. Porém, em condições de hipoxia, o feto tem uma acentuação de tais movimentos respiratórios (gasping), fazendo com que este material indesejado chegue até bronquíolos terminais e alvéolos ${ }^{(18)}$. Por outro lado, a conseqüente acidose leva a liberação esfincteriana e liberação de mecônio, o qual pode ser aspirado ${ }^{(52)}$. Admite-se que a simples presença de algumas escamas córneas no interior de alvéolos não tenha significado patológico, mas apenas traduza um episódio de hipoxia intra-uterina. Mesmo que grandes quantidades de líquido amniótico ou mecônio sejam aspiradas, a morte intra-uterina não será conseqüente a elas, mas, sim, pela hipoxia/anoxia desencadeante, muito embora o mecônio seja irritante, podendo levar a repercussões pulmonares importantes ${ }^{(53,54)}$. Deve-se lembrar, também, que o mecônio é capaz de produzir calcificação abdominal em casos de peritonite meconial ${ }^{(55,56)}$, hiperplasia do epitélio amniótico e degeneração da parede muscular de vasos umbilicais, quando presentes no líquido amniótico por um certo tempo ${ }^{(57)}$, demonstrando seu potencial irritativo e lesivo. No neonato, entretanto, a aspiração de líquido amniótico, com ou sem mecônio, é extremamente ruim, por levar a asfixia em um recém-nascido já em hipóxia. Se maciça, a morte sobrevém em questão de minutos. Quando em menor intensidade, a criança pode sobreviver, mas, em geral, desenvolve um quadro importante de doença da membrana hialiana, muitas vezes com pneumonia superimposta.

A autópsia de casos de aspiração maciça de líquido amniótico ou mecônio mostra um pulmão túrgido, contendo o material aspirado na luz traqueobrônquica e na superfície de corte, mostrando que o mesmo foi levado distalmente pela respiração ou manobras de ventilação. Se a aspiração foi menos intensa e a criança sobreviveu mais tempo, o que se observam são os sinais de membrana hialina ou pneumonia. É importante ressaltar que, durante a autópsia de qualquer recém-nascido, devem-se examinar os condu- 
tos auditivos, pois, muitas vezes, aí pode ser claramente encontrado o mecônio, se a criança porventura tiver sido previamente limpa ${ }^{(27 b)}$.

Do ponto de vista histológico, as aspirações maciças mostram grande quantidade de material estranho, contendo escamas córneas no interior de bronquíolos e alvéolos, algumas vezes formando verdadeiras rolhas. Nas aspirações de menor intensidade, podem ser encontradas as escamas córneas, associadas a membranas hialinas ou pneumonia.

Felizmente, ocorrências como esta são menos freqüentes em função da melhoria da atenção prénatal e obstétrica.

\section{Atelectasia}

Atelectasia pode ser definida como área de colapso alveolar pela inexistência de ar. Ela pode ser primária, como resultado da não expansão do pulmão fetal, ou secundária, conseqüente à absorção do ar contido nos espaços respiratórios sem que haja reposição dos mesmos, como nos casos obstrutivos, ou ain- da pela compressão pulmonar, como ocorre nos derrames pleurais, pneumotórax, cardiomegalia, massas tumorais, etc. Na faixa etária aqui abordada, pode ser resultante da não expansão pulmonar vista nos natimortos e neomortos, que não apresentaram movimentos respiratórios. A forma obstrutiva ocorre mais freqüentemente pela aspiração de mecônio, enquanto que a compressiva surge nos casos de pneumotórax ou de hidrotórax. A DMH está associada a atelectasia, pois a falta de surfactante leva a colapso alveolar. Pode ser vista, também, nas pneumonias e na displasia broncopulmonar.

As áreas atelectásicas apresentam-se macroscopicamente deprimidas e com uma coloração vinhosa mais intensa. Quando acometem um lobo o mesmo afunda completamente na água ao ser isolado. Histologicamente, aparecem como áreas sólidas, quando, ocasionalmente, podem ser confundidas com espessamento dos septos alveolares. Nos casos de pneumonia, DMH e DBP as alterações características destes quadros podem ser vistas em áreas vizinhas.

PERES LC; MORAES FA \& YUKITA CM. Contribution to the study of pulmonary pediatric pathology in autopsies. Medicina, Ribeirão Preto, 32: 303-315, july/sept. 1999

ABSTRACT: Study model: retrospective study. Objectives: The aim of the present study was to determine the frequency of some of the main pediatric pulmonary diseases among the autopsies performed at the Serviço de Patologia do HCFMRP-USP, compared to the pertinent literature in order to compose an updated text which could help mainly undergraduate students in understanding such problems. Methodology: The following data retrieved from the files of 505 pediatric autopsies performed between June 1993 and June 1996 were analyzed: sex, gestational age, age at death, body weight, cause of death, weight and pulmonary problems (petechiae, hemorrhage, lobation defects, hypoplasia, pneumonia, pneumothorax, hemothorax, pleural effusion, interstitial emphysema, hyaline membrane disease, bronchopulmonary dysplasia and atelectasia). The Epi-Info-6 program was used to tabulate and analyze the data. Results: Males predominated over female and indeterminate sex $(53.7 \% ; 45.9 \%$ and $0.4 \%$, respectively). Most of the deaths were observed in the early neonatal (47\%), intra-uterine (19\%) and late neonatal $(7 \%)$, periods, performing $73 \%$ of all cases and gestational age ranged from 16 to 43 weeks. Prematurity was the most frequent cause of death $(29.7 \%)$, followed by congenital anomalies $(10.9 \%)$, intrauterine anoxia $(8.3 \%)$, infections $(8.1 \%)$, perinatal causes $(2.4 \%)$, isoimmunization $(1.8 \%)$, neoplasia $(0.4 \%)$ and other causes $(38.4 \%)$. The pulmonary problems found were petechiae $(49.1 \%)$, massive hemorrhage (34.5\%), pleural effusion (29.5\%), hyaline membrane disease $(25.6 \%)$, atelectasia $(24.6 \%)$, pneumonia $(16.8 \%)$, pneumothorax (10.8\%), lobation defects $(8.6 \%)$, interstitial emphysema (5\%), hypoplasia $(4.4 \%)$ and bronchopulmonary dysplasia $(2.4 \%)$. Conclusions: Pulmonary problems are very common in pediatric autopsies and frequently represent the cause of death. Their frequency is similar to that reported in the pertinent literature and the variations possibly reflect the differences in pre and perinatal care in the different countries. Most of the pulmonary alterations found are linked to prematurity and congenital anomalies, corresponding to a third of the cases. Reduction of the frequency of these groups would have an important medical and socio-economic impact.

UNITERMS: Pediatrics. Lung Diseases. 


\section{REFERÊNCIAS BIBLIOGRÁFICAS}

1 - SILVA AR \& PERES LC. Levantamento de 1419 autópsias consecutivas em um hospital terciário. (Resumo) J Bras Patol 35:173, 1999. Suppl.

2 - MARIA E SILVA J \& MANÇO ARX. Aspectos epidemiológicos da mortalidade infantil em Ribeirão Preto. Informativo Epidemiológico de Ribeirão Preto - IERP, Núcleo de Vigilância Epidemiológica - HCFMRP-USP e Divisão de Vigilância Epidemiológica - Secretaria Municipal da Saúde - Ribeirão Preto, Ano II, no. 24, Dezembro/1998.

3 - KALOUSEK GK \& GILBERT-BARNESS E. Causes of stillbirth and neonatal death In: GILBERT-BARNESS E, ed., Potter's pathology of the fetus and infant Mosby-Year Book, St Louis, Chap. 5, v. I, p.128-162, 1997

4 - PERES LC; FERRAZ VEF; ACOSTAAC; ROJAS MVM; NOCE TR \& PINA NETO JM. Estruturação de um serviço de patologia pediátrica. A importância do trabalho conjunto entre genética médica e patologia. J Anátomo-Patol; 11:5-6, 1996

5 - KALBERG P \& ERICSON A. Perinatal mortality in Sweden. Analysis with international aspects. Acta Paediatr Scand 275:28-34, 1979. (Suppl)

6 - ÖSTÖR AG; STILLWELL R \& FORTUNE DW. Bilateral pulmonary agenesis. Pathology 10:243-248, 1978

7 - DEVI B \& MORE JR. Total tracheopulmonary agenesis associated with asplenia, agenesis of umbilical artery and other anomalies. Acta Paediatr Scand 55:107-116, 1996.

8 - ASKENAZI SS \& PERLMAN M. Pulmonary hypoplasia: lung weight and radial alveolar count as criteria of diagnosis. Arch Dis Child 54:614-618, 1976.

9a - COONEY TP \& THURLBECK WM. The radial alveolar count method of Emery and Mithal: a reappraisal 2 - Postnatal lung growth Thorax 37:580-5583, 1982.

9b - COONEY TP \& THURLBECK WM. The radial alveolar count method of Emery and Mithal: a reappraisal 1 - Postnatal lung growth Thorax 37:572-579, 1982.

10 - HISLOP A; HEYE E \& REID L. The lungs in congenital bilateral agenesis and dysplasia. Arch Dis Child 54:32-38, 1979.

11 - GAU GS. The respiratoty system In: KEELING JW, ed. Fetal and neonatal pathology Springer-Verlag, Berlin Heildelber, Chap. 18, p 363- 372-379, 1987.

12 - ASKIN F. Respiratory tract disorders in the fetus and neonate In: WIGGLESWORTH JS \& SINGER DB, ed. Textbook of fetal and perinatal pathology. Blackwell Scientific Publications, Oxford, Chap. 19, p 643-688 1991.

13 - GILBERT-BARNESS E. Respiratory system. In: GILBERTBARNESS E, ed, Potter's pathology of the fetus and infant, Mosby-Year Book, St. Louis, Chap. 19, v. 1, p 712773, 1997

14 - EMERY JL \& MITHAL A. The number of alveoli in the terminal respiratory unit of man during late intrauterine life and childhood. Arch Dis Child 35:544-547, 1960.
15 - LANDING BH; LAWRENCE TYK; PAYNE JR VC \& WELLS TR. Bronchial anatomy in syndromes with abnormal visceral situs, abnormal spleen and congenital heart disease. Am J Cardiol 28:456-462, 1971.

16 - LANDING BH. Five syndromes (malformation complexes) of pulmonary symmetry, congenital heart disease and multiple spleens. Summary and conclusions. Pediatr Pathol 2:148-151, 1984.

17 - DIEHL EJ \& LANDING HB. Syndrome of pulmonary isomerism of right lung type, congenital disease, pulmonary and systemic venous abnormalities and malrotaion of intestine, with a spleen or multiple spleens ( $m$-aniosplenia): comparison with Ivemark asplenia syndrome. Pediatr Pathol 2:133138, 1984.

18 - COX JN. Respiratory system. In: BERRY CL, ed, Paediatric pathology. 2nd ed. Springer-Verlag, Berlin Heildelber, Chap. 7, p 293-407, 1989.

19 - STOCKER JT; MADEWELL JE \& DRAKE RM. Congenital cystic adenomatoid malformation of the lung. Classification and morphologic spectrum. Hum Pathol 8:155-171 , 1977

20 - STOCKER JT. Malformation of the distal pulmonary acinus: congenital cystic adenomatoid malformation - type 4, a report of 12 cases (Abstract), Pediatr Pathol 15:313, 1994

21 - RUTLEDGE JC \& JENSEN P. Acinar dysplasia: a new form of pulmonary maldevelopment. Hum Pathol 17:1290-1293, 1986.

22 - MOESSINGER AC; DRISCOLL JR JM \& WIGGER HJ. High incidence of lung perforation by chest tube in neonatal pneumothorax. J Pediatr 92:635-637, 1978.

23 - LINDROTHM; SVENNINGSEN NW; AHLSTROM H \& JONSONB. Evaluation of mechanical ventilation in newborn infants. I. Techniques and survival rates. Acta Paediatr Scand 69:143149, 1980.

24 - WOOD B; ANDERSON VM; MAUK JE \& MERRITT TA. Pulmonary lymphatic air: locating "Pulmonary Interstitial Emphysema" of the premature infant. Am J Radiol 138:809-814, 1982.

25 - OPPERMANN HC; WILLE L; OBLADEN M \& RICHTER E.MSystemic air embolism in the respiratory distress syndrome of the newborn. Pediatr Radiol 8:139-145, 1979.

26 - CHESSELLS JM \& WIGGLESWORTH JS. Haemostatic failure in babies with Rhesus isoimmunization. Arch Dis Child 46:38-45, 1971.

27a - KEELING JW. Fetal hydrops In: KEELING JW, ed, Fetal and neonatal pathology Springer-Verlag, Berlin Heildelber, Chap. 11, p 211-228, 1987

27b - KEELING JW. Intrapartum asfixia and birth trauma In: KEELING JW, ed, Fetal and neonatal pathology SpringerVerlag, Berlin Heildelber, Chap. 10, p 199- 210, 1987.

28 - LARROCHE JC. Anasarque foeto-placentaire (hydrops) sans immunisation. Med Hyg 40:2061-2076, 1982.

29 - BERRY PJ; GRAY ES; PORTER HJ \& BURTON PA. Parvovírus infection of the human fetus and newborn. Semin Diagn Pathol 9:4-12, 1992. 
30 - ISFER EV; SANCHES RC \& SAITO M. Diagnóstico pré-natal: conduta e tratamento. In: BITTENCOURT AL. Infecções congênitas transplacentárias. Revinter, Rio de Janeiro, p 93-123, 1995.

31 - PERES LC; CUNHAMERCANTE AM \& HIROKAWAZT. Fibrose pelural em fetos abortados J Bras Patol 33:76-83, 1997.

32 - REYNOLDS EOR; ROBERTON MB \& WIGGLESWORTH JS. Hyaline membrane disease, respiratory distress and surfactant deficiency. Pediatrics 42:758-767, 1968.

33 - SCHOFIELD D \& COTRAN RS. Diseases of infancy and childhood In: COTRAN RS; KUMAR V \& COLLINS T, ed, Robbins' pathologic basis of disease, W. B. Saunders, Philadelphia, Chap. 11, p 459- 491, 1999.

34 - FARRELL PM \& AVERY ME. Hyaline membrane disease. Am Rev Resp Dis 111:657-679, 1975.

35 - BOURBON JR \& FARRELL PM. Fetal lung development in the diabetic pregnancy. Pediatr Res 19:253-263, 1985.

36 - FADEL HE; SAAD AS; NELSON GH \& DAVIS HC. Effect of maternal-fetal disorders on lung maturation. I. Diabetes meliitus. Am J Obstet Gynecol 155:544-553, 1986.

37 - BALLARD PL; HAWGOOD S, LILLEY H, WELLENSTEIN G, GONZALES LW, BENSON B, CORDELL B \& WHITE T. Regulation of pulmonary surfactant apoprotein SP 28-36 gene in fetal human lung. Proc Nat Acad Sci USA 83:9527-9531, 1986.

38 - BALLARD RA \& BALLARD PL Use of prenatal glucocorticoid therapy to prevent respiratory distress syndrome. A supporting view. Am J Dis Child 130:982-987,1976

39 - BALLARD PL \& BALLARD RA Glucocorticoids in prevention of respiratory distress syndrome. Hosp Pract 15:81-87,1980

40 - BALLARD PL \& BALLARD RA Scientific basis and therapeutic regimens for use of antenatal glucocorticoids. Am J Obstet Gynecol 173:254-262,1995

41 - JOBE AH. Pulmonary surfactant therapy. N Engl J Med 328:861-866, 1993.

42 - O'BRODOVICH HM \& MELLINS RB. Bronchopulmonary dysplasia. Unresolved neonatal acute lung injury. Am Rev Resp Dis 132:694-709, 1985

43 - LEE RMKW \& ROSSMAN CM. Cilliary defects associated with the development of bronchopulmonary dysplasia. Am Rev Resp Dis 129:190-193,1984.

44 - MERRITT TA. Oxygen exposure in the newborn guinea pig lung lavage cell populations, chemotatic and elastase response: a possible relationship to neonatal bronchopulmonary dysplasia. Pediatr Res 16:798-805, 1982.
45 - COLE VA; NORMAND ICS; REYNOLDS EOR \& RIVERS RPA Pathogenesis of hemorrhagic pulmonary edema and massive pulmonary hemorrhage in the newborn. Pediatrics 51:175-186, 1973.

46 - KROUS HF \& JORDAN J. A necropsy study of distribution of petechiae in non-sudden infant death syndrome. Arch Pathol Lab Med 108:75-76, 1984.

47 - BERRY PJ. Pathological findigns in SIDS. J Clin Pathol 45:11 $16,1992$.

48 - MAYO P \& SAHA SP. Spontaneous pneumothorax in the newborn. Am Surgeon 49:192-195, 1983.

49 - MADANSKY DH; LAWSON EE; CHERNICK V \& TAEUSCH Jr W. Pneumothorax and other forms of pulmonary air leak in newborns. Am Rev Resp Dis 120:729-737, 1979.

50 - RUDD PT \& WIGGLESWORTH JS. Oxygen embolism during mechanical ventilation with disapeearence of signs after death. Arch Dis Child 57:237-239, 1982.

51 - PATRICK J; CAMPBELL K; CARMICHAEL L, NATALE R \& RICHARDSON B. Patterns of human breathing during the last 10 weeks of pregnancy. Obstet Gynecol 56:24-30, 1980.

52 - VIDYASAGAR D; HARRIS V \& PILDES RS. Assisted ventilation in infants with meconium aspiration syndrome. Pediatrics 56: 208-213, 1975.

53 - BYRNE DL \& GAU GS. In utero meconium aspiration: an unpreventable cause of neonatal death. $\mathbf{B r} \mathbf{J}$ Obstet Gynecol 94:813-814, 1987.

54 - DAVIS RO; PHILIPS JB $3^{\text {rd }}$; HARRIS Jr BA; WILSON ER \& HUDDLESTON JF . Fatal meconium aspiration syndrome occurring despite airway manegement considered appropriate. Am J Obstet Gynecol 151:731-736, 1985.

55 - RICKHAM PP. Peritonitis in the neonatal period. Arch Dis Child 30:23-31, 1955.

56 - WHITE RB. Meconium peritonitis: a surgical emergency. J Pediatr 48:793-796, 1956.

57 - ALTSHULER G \& HYDE S. Meconium induced vasoconstriction: a potencial cause of cerebral and other fetal hypoperfusion and of poor outcome. J Child Neurol 4: 137-142, 1989.

Recebido para publicação em 18/06/99

Aprovado para publicação em 14/09/99 